\title{
Medical dispute resolution, patient safety and the doctor-patient relationship
}

\author{
Kumaralingam Amirthalingam, LLB(Hons), $\mathrm{PhD}$
}

\section{INTRODUCTION}

This paper argues that most medical disputes are better resolved through alternative dispute resolution mechanisms and that these mechanisms can contribute to improving patient safety by encouraging more candid and comprehensive reporting of risks. It also argues that medical disputes and patient safety have to be viewed through a new lens, namely patient autonomy. Autonomy has come to dominate the discourse on medical negligence in recent years. It reached a high watermark in the recent landmark United Kingdom Supreme Court decision of Montgomery v Lanarkshire Health Board, ${ }^{(1)}$ in which the Supreme Court, overruling its earlier jurisprudence on the medical duty to inform, endorsed the Australian Rogers $v$ Whitaker ${ }^{(2)}$ test of the prudent patient in preference to the conventional test of the reasonable doctor, established in Bolam v Friern Hospital Management Committee. ${ }^{(3)}$ The court in Montgomery recognised that the doctor-patient relationship had fundamentally changed, with patients now much better informed and actively involved in making decisions affecting their bodily integrity and personal autonomy.

This new reality sometimes creates a tension between patient autonomy and patient safety, and it is important explicitly to recognise this tension. One of the main causes of medical disputes is patients' resentment that they are not given sufficient information in order to make their own decisions. Litigation is resorted to not just for compensation but for patients to get more information about what actually happened to them. Thus, it is important to disentangle questions of patient safety (which requires a systemic approach affecting processes and protocols) from questions of medical dispute resolution (which focuses on the particular facts and relationship of the parties).

\section{PATIENT AUTONOMY}

The vast majority of medical disputes arise out of medical negligence. The plaintiff must prove that the defendant owed a duty of care, breached that duty and caused damage that is not too remote. However, each of these aspects of negligence becomes more complex in medical malpractice suits. To whom is the duty owed? Is it to the patient, the unborn child, the spouse, the employer, the parents, or the stranger in distress? What is the scope of that duty? Is it to prevent physical injury, psychological harm, emotional distress, pure economic loss, expectations loss, loss of chance, or loss of autonomy?
How is the standard of care to be determined? The relationship between doctor and patient is not like the relationship between pedestrian and motorist. There is a prior relationship of trust, vulnerability and expectation. The medical choices are infinite and doctors have to take into account not just the patients' emotions, idiosyncrasies and autonomy, but also the institutional culture of the medical services provider as well as the practices and policies of insurers and managed care systems. Causation in medical negligence is especially complex. The trial process, which is adversarial, bound by strict rules of evidence and usually takes place years after the event, is not the best mechanism to determine liability, let alone understand what actually happened.

2016 is a big year for medical negligence in Singapore and Malaysia. The Federal Court of Malaysia is set to hear at least five appeals in December to determine, among other things, the Bolam/Rogers test for the standard of care, and the extent of a hospital's vicarious liability and non-delegable duties with respect to medical negligence. In Singapore, there are two appeals before the Court of Appeal arising out of the High Court decisions in Hii Chii Kok v Ooi Peng Jin London Lucien and another ${ }^{(4)}$ and Thomson Medical Centre v ACB. ${ }^{(5)}$ In Hii Chi Kok, the Court of Appeal has been invited to reconsider the standard-of-care test with respect to the duty to inform, i.e. whether it should continue with the classic Bolam test or adopt the Montgomery test.

In Montgomery, the infant child suffered from cerebral palsy during childbirth. The mother, who was diabetic, was carrying a child that was larger than normal. The mother herself was quite small, increasing the risk of shoulder dystocia during vaginal delivery, as the child's shoulder might not be able to pass through the pelvis. The risk materialised with tragic consequences. It was alleged that the doctor was negligent in failing to inform the mother of the risk of vaginal delivery and in failing to perform an emergency Caesarean. In finding the doctor liable, Lady Hale, in her concurring opinion, emphasised the significance of patient autonomy: "it is now well recognised that the interest which the law of negligence protects is a person's interest in their own physical and psychiatric integrity, an important feature of which is their autonomy, their freedom to decide what shall and shall not be done with their body (emphasis added)."16

In Thomson Medical Centre, the Court of Appeal is grappling with complex questions about damages in a "wrongful birth" type case. The plaintiffs (husband and wife) had a child through IVF. There was a mix-up in the donor's sperm due to the negligence 
of the defendant, resulting in the conception of a baby who was not biologically that of the husband. The question is whether the parents are entitled to claim damages for the cost of raising the child. This raises complex legal and moral questions with the international jurisprudence divided: some countries allow such claims and others deny them. However, even in the UK, which denies such claims, the House of Lords recognised that in these cases, the autonomy interests of the parents have been adversely affected, meriting some compensation. Again, although from a different perspective, patient autonomy is central to medical negligence discourse.

The Singapore Medical Council, in its revised guidelines issued earlier this year, highlights the importance of patient autonomy both in its foreword and within the guidelines. Section C5 states explicitly, "Patient autonomy is a fundamental principle in medical ethics and must be respected."

C5(3) is as follows: you must ensure that patients are made aware of the purpose of tests, treatments or procedures to be performed on them, as well as the benefits, significant limitations, material risks (including those that would be important to patients in their particular circumstances) and possible complications as well as alternatives available to them (emphasis added).

This paragraph reflects the Montgomery/Rogers disclosure standard based on material risk. Patient autonomy is a recurrent theme affecting medical malpractice today and it has to be taken into account both in resolving medical disputes and in developing patient safety protocols.

\section{PATIENT SAFETY}

Does medical litigation improve patient safety? There is no evidence to support an unequivocal affirmative answer. What is clear is that medical litigation is costly and ineffective. Twenty years ago, Lord Woolf, in his 1996 Access to Justice Report, stated, "early in the Inquiry it became increasingly obvious that it was in the area of medical negligence that the civil justice system was failing most conspicuously to meet the needs of litigants in a number of respects." ${ }^{\prime(7)}$ The Report noted that the costs were disproportionately high and that there were significant delays in resolving claims. Often, unmeritorious claims were pursued by disgruntled patients while clear-cut claims were vigorously defended by medical insurers.

This is supported by United States data showing that over $60 \%$ of medical malpractice claims are summarily dismissed as having no merit. ${ }^{(8)}$ On the other hand, based on data from the Harvard Medical Practice Study of 1991, researchers estimated that only $2 \%$ of patients injured through medical negligence actually sue. ${ }^{(8,9)}$ It seems that many who should not sue are doing so while many who should are not. The Woolf Report further noted that the success rate for medical negligence litigation was lower than in other personal injury litigation. Again, US data supports this, with research showing that doctors prevail in about $90 \%$ of the cases in which the evidence of medical negligence is weak and in about half the cases where the evidence of medical negligence is strong. ${ }^{(10)}$ Finally, the Woolf Report noted that suspicion and animosity between parties in medical negligence litigation is generally higher than in other areas of litigation.

The answer to the question of whether litigation raises professional standards and patient safety is that it most likely does not. Anecdotal evidence suggests that doctors either settle claims with a non-disclosure agreement, or if they do not believe they are personally at fault, take their chances with litigation where they have a high chance of successfully defending the action. This does not encourage a culture of sharing information and learning from mistakes. Litigation is a compensation lottery with no proven impact on patient safety, but with increasing evidence that it contributes to defensive medicine and prevents reporting of adverse incidents. Separate strategies are required to improve patient safety and to resolve medical disputes.

Patient safety is improved through systemic intervention rather than by identifying individual instances of negligence. In 1999, the Institute of Medicine (now the Academy of Medicine) released a groundbreaking report, To Err is Human: Building a Safer Health System. ${ }^{(11)}$ The report caused a stir, as it highlighted the significant number of preventable deaths and injuries due to adverse events in hospitals. It estimated the occurrence of preventable deaths at between a conservative estimate of 44,000 and an outer estimate of 98,000 per year, far exceeding annual deaths due to traffic accidents. These eye-catching figures have, however, been doubted and a recent British study suggests that many of these early studies overestimated the number of preventable deaths. ${ }^{(12)}$ Leaving aside the numbers, the To Err is Human report argued that the way to prevent or reduce adverse outcomes was to focus on patient safety rather than on professional negligence. It advocated systemic change to prevent medical mishaps, stating, "one of the report's main conclusions is that the majority of medical errors do not result from individual recklessness or the actions of a particular group--this is not a "bad apple" problem. More commonly, errors are caused by faulty systems, processes, and conditions that lead people to make mistakes or fail to prevent them". ${ }^{(13)}$

Focusing on individual liability instead of systemic accountability as a strategy to improve patient safety is akin to favouring hook-and-line fishing over net fishing to feed the masses. This is not to say that doctors should never be held personally responsible, but a greater distinction should be drawn between medical errors and medical negligence. ${ }^{(14)}$ By removing personal blame, there is greater incentive for doctors to report errors and improve patient safety.

If litigation does not contribute to improving patient safety, why is it necessary? It remains necessary because it is vital that individuals have access to courts to seek justice; the trial process, for all its weaknesses, does provide procedural fairness and legitimacy. Most importantly, it is open and transparent. However, for the most part, litigation probably does more harm than good in resolving medical disputes and alternative resolution for the majority of disputes is preferable. Litigation is designed to result in a win-lose outcome, as one medical negligence litigator graphically illustrates using a war metaphor: "litigation is based upon a war model. The parties muster an army (the firm), appoint 
a general (the lead trial lawyer), choose a battleground (the court with jurisdiction), stockpile the ammunition (discovery) and engage in battles (the motion practice), participate in a required peace effort (pre-trial settlement conference), blow each other to bits (trial) and declare a victor (the verdict)" ${ }^{1{ }^{(15)}}$

The question is whether alternative dispute resolution (ADR) can provide a win-win outcome.

\section{ALTERNATIVE DISPUTE RESOLUTION}

Medical disputes often arise - or at least escalate - because of poor communication. Recently, it was reported that one in five complaints against doctors in Singapore arises out of poor communication. ${ }^{(16)}$ Studies show that in many cases, the main reason a patient sues a doctor is not to seek compensation but to find out what went wrong. Litigation does not encourage open communication or disclosure. Would ADR mechanisms fare any better? ADR refers to resolving disputes without resorting to litigation. It includes negotiation, mediation and arbitration.

Historically seen as an alternative to litigation, ADR is increasingly being used in conjunction with litigation as a screening or settlement device. Chief Justice Menon, in his opening address at the Global Pound Conference Series held in Singapore in March this year, outlined a holistic approach to dispute resolution. ${ }^{(17)}$ Instead of viewing arbitration and mediation as 'alternative' dispute resolution, he championed what he termed 'appropriate' dispute resolution. Rather than viewing ADR as an alternative to litigation, courts should promote a combination or hybrid mechanism that works best for the particular dispute.

The general arguments in favour of ADR - especially mediation - are that they promote conciliation, facilitate creative settlements and enable the parties to present explanatory rather than defensive narratives. An experienced American medical negligence mediator describes it as providing a "therapeutic resolution" of the dispute, allowing parties an opportunity to explain or receive an explanation, to apologise or to forgive, and to have closure and to restore relationships. This sounds idealistic, and indeed, there is a danger of romanticising mediation.

Mediation is particularly suited to disputes where the parties have an interest in an ongoing relationship. A classic example comes from family law, where divorcing parents have a mutual interest in managing their relationship to deal with child custody issues and maintenance. However, in medical disputes, often the relationship has actually ended, so there is no interest in continuing or repairing it. ${ }^{(18)}$ Patients want an explanation and compensation, while doctors - and the institutions with which they are affiliated - want to protect their reputations, and financial interests. Mediation can help reframe the medical dispute away from the war-footing and help preserve the relationship. This can lead to better outcomes for both doctor and patient, in terms of resolving the dispute and improving patient safety. ${ }^{(19)}$

Medical disputes have unique features that make them particularly challenging for mediation. First, they often involve complex medical facts and issues of causation, making it difficult for an untrained mediator. Secondly, medical disputes can involve a range of parties: the slew of medical personnel from referring doctor to consultant to nurse, the insurer, the hospital management, the patient or the next of kin and the lawyer. Each has a different perspective, based on their professional training or institutional culture, making a meeting of minds challenging. Thirdly, there is a power imbalance in medical disputes between the healthcare provider and the patient. Fourthly, the confidentiality and privacy issues are unlike those in other disputes due to the sensitivity of health records and the personal nature of the information.

Perhaps, the two biggest obstacles to mediation of medical disputes come from the key players - doctors and lawyers. ${ }^{(20)}$ American studies of medical mediation show that most mediation takes place without the doctor being present for a variety of reasons: they do not want to face their patients; they are too busy with their practice; or they just want to leave it to their insurers to settle. Often, defence lawyers tell doctors not to attend mediation for fear that doctors may be too candid, leaving them vulnerable if mediation fails and the patient proceeds with litigation. Apart from discouraging doctors from attending mediation, some lawyers do not like it because mediation affects their economic interests. Mediations are much quicker, affecting the number of hours they can bill. One US study showed that a lawyer spent 3.5 hours on average preparing for mediation and over 36 hours preparing for trial. ${ }^{(21)}$

Given these inherent difficulties, how can mediation be used more widely to resolve medical disputes? There are no easy answers, but the first step is to make a commitment to genuine mediation, and not merely to mediation as a means or a guise for settlement or to test the waters for litigation. At the heart of mediation is the notion that the parties own the dispute and that they are willing to work with each other to reach a mutually acceptable outcome. Yet, mandatory mediation schemes often do not have these features. Indeed, many mandatory schemes arguably are not genuine forms of mediation. ${ }^{(15)}$ As one mediator put it, these "processes are called mediation even when there is little or no relationship with mediation theory and practice. When the programme fails, mediation gets the blame rather than some other process." ${ }^{\prime(22)}$

There are a few basic principles for successful medical mediation: ${ }^{(15,22)}$

- $\quad$ Self-determination - this means the parties must be willing and voluntary participants, and they must be able to leave the mediation at any time. This does not necessarily preclude mandatory mediation; as one mediator observed, mandatory medical mediation is necessary to get the parties there, but once they are there, they must be free to leave at any time.

- Impartiality - the mediator must be impartial in order for the parties to have trust and for the process to have credibility. In mediation, there is a power imbalance as the defence is often a repeat player and has an advantage. The mediator has to protect the patient's interest while guarding against becoming an advocate for the patient.

- $\quad$ Flexibility - the solutions have to fit the particular dispute and the expectations of the parties. The mediator needs to be creative and parties must be willing to explore different 
solutions. Examples include apologies, donations to charities, free medical care and education of children.

- Confidentiality - it is vital that whatever is said during mediation remains confidential; otherwise, it will be difficult to have a full and frank discussion, as the parties will worry that what they say may be used in court if mediation fails.

\section{CONCLUSION}

Patient safety requires a systems approach with attention to institutional, rather than individual, players. The literature on institutional liability highlights the relevance of institutional culture and the constraints on individuals acting within that institutional culture. ${ }^{(23)} \mathrm{A}$ case in point is the recent spotlight on police shootings in the US. Do we blame the individual police officer or do we examine the policing culture to prevent such incidents from happening?

Medical disputes require respect for patient autonomy and treating the patient as an equal partner in managing his or her health issues. Giving opportunities for the individuals on either side of the dispute to present their narratives in a non-confrontational environment is healthy. Common to both systemic strategies to improve patient safety and personalised strategies to deal with dispute resolution are good communication and transparency, which are critical to identifying risks in the healthcare system and to preserving the trust inherent in the doctor-patient relationship.

\section{POSTSCRIPT}

This revised text was completed at the end of October 2016. Since then, judgment in the two Court of Appeal cases mentioned in the lecture, namely, Thomson Medical Centre and Hii Chii Kok, have been delivered. ${ }^{(24,25)}$ In both cases, the Court of Appeal reiterated the centrality of patient autonomy to the doctor-patient relationship. Briefly, in Thomson Medical Centre, the Court of Appeal, recognising loss of genetic affinity as a new form of actionable damage, awarded substantial damages to vindicate the claimant's autonomy interest. In Hii Chii Kok, the Court of Appeal adapted the Montgomery test for the duty to inform, holding that the materiality of a risk had to be determined from the perspective of the patients in order to respect their autonomy.

\section{REFERENCES}

1. [2015] AC 1430 .

2. [1992] 175 CLR 479.

3. [1957] 1 WLR 582

4. [2016] 2 SLR 554.

5. [2015] 2 SLR 218.

6. Montgomery $v$ Lanarkshire Health Board [2015] AC 1430 at [108].

7. Lord Woolf. Access to Justice: Final Report to the Lord Chancellor on the Civil Justice System in England and Wales (London: HMSO, 1996) ch 15, para 2.

8. Sohn DH, Bal BS. Medical malpractice reform: the role of alternative dispute resolution. Clin Orthop Relat Res 2012; 470:1370-8.

9. Localio AR, Lawthers AG, Brennan TA, et al. Relation between malpractice claims and adverse events due to negligence. Results of the Harvard Medical Practice Study III. N Engl J Med 1991; 325:245-51.

10. Peters PG Jr. Twenty years of evidence on the outcomes of medical malpractice claims. Clin Orthop Relat Res 2009; 467:352-7.

11. To Err is Human: Building a safer health system. In: Institute of Medicine, National Academy of Sciences [online]. Available at: http://www. nationalacademies.org/hmd/ /media/Files/Report\%20Files/1999/To-Err-isHuman/To\%20Err\%20is\%20Human\%201999\%20\%20report\%20brief.pdf. Accessed October 27, 2016.

12. Hogan $H$, Healey $F$, Neale G, et al. Preventable deaths due to problems in care in English acute hospitals: a retrospective case record review study. BMJ Qual Saf 2012; 21:737-45.

13. Kohn LT, Corrigan JM, Donaldson MS, eds. To Err is Human: Building a Safer Health System. Washington, DC: National Academy Press, 1999.

14. Marx D. Patient safety and the 'just culture': a primer for health care executives. In: Medical Event Report System - Transfusion Medicine (MERS-TM) [online]. Available at: http://www.safer.healthcare.ucla.edu/safer/archive/ahrq/ FinalPrimerDoc.pdf. Accessed October 18, 2016.

15. Johnson SM. A medical malpractice litigator proposes mediation. Dispute Res J 1997; 52.

16. Lai L. 1 in 5 complaints against doctors due to poor communication. The Straits Times 2016 Sep 18.

17. Menon S. Shaping the future of dispute resolution and improving access to justice. In: Global Pound Conference Series 2016 - Singapore. Available at: http://www.supremecourt.gov.sg/Data/Editor/Documents/[Final]\%20 Global\%20Pound\%20Conference\%20Series $\% 202016 \% 20-\% 20$ 'Shaping\%20 the $\% 20$ Future $\% 20$ of $\% 20$ Dispute $\% 20$ Resolution $\% 20 \% 20$ Improving $\% 20$ Access\%20to\%20Justice'.pdf. Accessed November 16, 2017.

18. Metzloff TB, Peeples RA, Harris CT. Empirical perspectives on mediation and malpractice. Law Contemp Probl 1997; 60:107-52.

19. Hyman CS, Liebman CB, Schechter CB, Sage WM. Interest-based mediation of medical malpractice lawsuits: a route to improved patient safety? J Health Polit Policy Law 2010; 35:797-828.

20. Bogdanoski T. Medical negligence dispute resolution: a role for facilitative mediation and principled negotiation? Australasian Disp Resol J 2009; 20:77-87.

21. Szmania SJ, Johnson AM, Mulligan M. Alternative dispute resolution in medical malpractice: a survey of emerging trends and practices. Conflict Resol Q 2008; 26:71-96.

22. Currie CM. Mediation and medical practice disputes. Conflict Resol Q 1998; 15:215-26.

23. Hall MI. Theorizing the Institutional Tortfeasor. Alberta L Rev 2016; 53:995-1011.

24. ACB v Thomson Medical Pte Ltd and others [2017] SGCA 20.

25. Hii Chii Kok v Ong Peng Jin London Lucien and another [2017] SGCA 38. 